\title{
Atrial Fibrillation Ablation without Interruption of Anticoagulation
}

\author{
Pasquale Santangeli, ${ }^{1}$ Luigi Di Biase, ${ }^{1,2,3}$ Javier E. Sanchez, ${ }^{1}$ Rodney Horton, ${ }^{1}$ \\ and Andrea Natale ${ }^{1,2,4,5,6,7}$
}

\author{
${ }^{1}$ Texas Cardiac Arrhythmia Institute, St. David's Medical Center, 1015 East 32nd Street, Suite 516, Austin, TX 78705, USA \\ ${ }^{2}$ Department of Biomedical Engineering, University of Texas, Austin, TX 78712, USA \\ ${ }^{3}$ Department of Cardiology, University of Foggia, Viale Pinto 1, 71100 Foggia, Italy \\ ${ }^{4}$ Division of Cardiology, Stanford University School of Medicine, Palo Alto, CA, USA \\ ${ }^{5}$ Case Western Reserve University, Cleveland, $\mathrm{OH}, \mathrm{USA}$ \\ ${ }^{6}$ Interventional Electrophysiology, Scripps Clinic, San Diego, CA, USA \\ ${ }^{7}$ EP Services, California Pacific Medical Center, San Francisco, CA, USA
}

Correspondence should be addressed to Andrea Natale, dr.natale@gmail.com

Received 7 November 2010; Accepted 24 February 2011

Academic Editor: Máximo Rivero-Ayerza

Copyright ( $\odot 2011$ Pasquale Santangeli et al. This is an open access article distributed under the Creative Commons Attribution License, which permits unrestricted use, distribution, and reproduction in any medium, provided the original work is properly cited.

Atrial fibrillation (AF) can be cured by pulmonary vein antrum isolation (PVAI) in a substantial proportion of patients. The high efficacy of PVAI is partially undermined by a small but concrete periprocedural risk of complications, such as thromboembolic events and bleeding. A correct management of anticoagulation is essential to prevent such complications. Performing PVAI without interruption of oral anticoagulation has been demonstrated feasible by our group in previous studies. Recently, we reported that continuation of therapeutic warfarin during radiofrequency catheter ablation consistently reduces the risk of periprocedural stroke/transient ischemic attack without increasing the risk of hemorrhagic events. Of note, interrupting warfarin anticoagulation may actually increase the risk of stroke even when bridged with heparin. The latter strategy is also associated with an increased risk of minor bleeding. With regard to major bleeding, we found no significant difference between patients with a therapeutic INR and those who were bridged with heparin. Therefore, continuation of therapeutic warfarin during ablation of AF appears to be the best anticoagulation strategy. In this paper we summarize our experience with AF ablation without interruption of anticoagulation.

\section{Introduction}

Atrial fibrillation (AF) is the most common sustained arrhythmia in Western countries, with an estimated 30 million patients affected by 2050 across United States and Europe alone [1]. Atrial fibrillation has a significant impact on morbidity mainly related to symptoms, heart failure, and thromboembolic events and is the most frequent arrhythmic cause of hospital admission in the USA [1-3]. In addition, AF is associated with excess mortality independently of thromboembolic complications [4]. To date, the most effective treatment for AF is radiofrequency catheter ablation, and pulmonary vein antrum isolation (PVAI) is the mainstay of such an approach [5]. The major drawback of catheter ablation of AF consists in its potential risk of periprocedural complications [6], with thromboembolic and hemorrhagic complications being among the most common and insidious ones $[7,8]$. Despite the introduction of novel ablation technologies, such as open irrigation catheters, and the widespread use of systemic anticoagulation with heparin, the risk of periprocedural thromboembolism remains not negligible, reaching up to $2.8 \%$ in large series $[6,9]$. With these premises, the development of novel strategies able to further decrease the risk of periprocedural thromboembolism without increasing the risk of bleeding is crucial.

Oral anticoagulant therapy with warfarin is the current standard of care for the treatment of AF, with a strikingly favorable balance between thromboembolic protection and 
bleeding complications when adequate monitoring of international normalized ratio (INR) is achieved [10]. For years, discontinuation of warfarin before ablation associated with the periprocedural use of unfractioned and low molecular weight heparin and bridging with warfarin after ablation has been the most widespread anticoagulation protocol in patients undergoing catheter ablation of $\mathrm{AF}[5,11]$. We firstly described the feasibility of AF ablation without interruption of oral anticoagulation [12] and recently reported that this approach is able to potentially abolish thromboembolic complications without increasing the risk of bleeding [13]. Accordingly, AF ablation without interruption of anticoagulation is nowadays the standard protocol in our center. In this paper, we summarize our approach to anticoagulation in patients undergoing $\mathrm{AF}$ ablation.

\section{Preprocedural Management}

Patients eligible for catheter ablation of AF are started on warfarin as outpatients, at least 2 months before the scheduled procedure. All patients receive weekly INR monitoring during the 4 to 6 weeks preceding the procedure, with a target INR of 2 to 3. Preprocedural transesophageal echocardiography (TEE) is performed only in patients showing subtherapeutic INR values in the month prior to the procedure. Patients who demonstrate INR values consistently above 2 in the month before the procedure are directly sent to ablation. We routinely do not discontinue therapeutic warfarin before the procedure.

\section{Periprocedural Management}

Ablation procedure is performed under general anesthesia. Four venous accesses are obtained: two right and one left femoral venous accesses, and one right internal jugular vein access. Femoral venous accesses are usually obtained with the Seldinger technique or with ultrasound guidance in difficult cases. The right internal jugular vein is accessed with ultrasound guidance or under fluoroscopic guidance with a wire or a deflectable catheter advanced into this vein via the right femoral vein sheath. A double transseptal puncture is performed with the assistance of intracardiac echocardiography (ICE) $[13,14]$. Before transseptal punctures, all patients receive a bolus of unfractionated heparin $(10,000$ Units), followed by a noncontinuous infusion to maintain an activated coagulation time (ACT) $>300$ seconds. We found important the addition of unfractionated heparin to periprocedural therapeutic warfarin, since soft thrombus can still be observed on the transeptal sheath or left atrial catheters in patients with periprocedural therapeutic INR.

Radiofrequency energy is delivered with an open irrigated ablation catheter with a maximum temperature of $42^{\circ} \mathrm{C}$, power up to $45 \mathrm{~W}$, and flow rate of $30 \mathrm{~cm}^{3} / \mathrm{min}$. Intracardiac echocardiography is continuously used to monitor the electrode surface during ablation, to assist with catheter positioning and identify coagulum formation, and to monitor for complications including pericardial effusion. An esophageal temperature probe is always inserted to assist with power titration during posterior wall ablation. At the end of the left atrial ablation, we partially reverse heparin anticoagulation with up to $40 \mathrm{mg}$ of protamine guided by the $\mathrm{ACT}$ and remove the sheaths when the ACT is less than 250 seconds.

With this approach there is no increased incidence of major bleeding complications compared with bridging therapy [13]. The majority of the major bleedings are related to cardiac tamponade, which can be effectively managed with emergent pericardiocentesis, together with heparin interruption and reversal with protamine, and warfarin reversal with fresh-frozen plasma or prothrombin complex concentrate [13]. All patients requiring pericardiocentesis are discharged on anti-inflammatory agents for two weeks. If continued significant drainage or reaccumulation of the pericardial effusion occurs despite these measures, emergent open surgical exploration is considered. Notably, there is also no difference in rates of emergent surgical exploration between patients with periprocedural therapeutic INR and those who discontinue warfarin, although patients on therapeutic anticoagulation are more likely to have a larger amount of blood removed from their pericardium for stabilization and require more blood transfusion units [13].

\section{Postprocedural Management}

All patients receive a single dose of aspirin $(325 \mathrm{mg}$ ) before leaving the electrophysiology laboratory and continue their warfarin dosage regimens to maintain a target INR of 2 to 3.

The beneficial effect of periprocedural therapeutic INR possibly extends also to the postprocedural period. After ablation the thromboembolic risk may be further increased by the procedure-related endothelial damage, which may activate the coagulation cascade and increase the risk of thromboembolism [15].

Therefore, warfarin discontinuation may be associated with an increased thromboembolic risk also in the postprocedural period, since reachieving a therapeutic INR after the procedure may take several days, and the risk of left atrial thrombosis during AF is strikingly time dependent.

All patients are strictly monitored for outcome and complications during overnight hospital stay, and on the following day prior to discharge using symptom assessment, serial neurological examinations, and puncture site checks. All patients are instructed to call in case of any symptom development and to send weekly transtelephonic electrocardiogram transmissions for the first 5 months after ablation. Progress of recovery and symptoms are assessed as well by a dedicated nurse. In case of symptoms or suspected complications patients are asked to seek medical attention at either a local emergency department or our emergency department or to follow up with their local physician. All documentations from these visits are collected by our AF center. Moreover, all patients present for followup 3 to 4 months after ablation with the electrophysiologist who performed the procedure.

With regard to the out-of-hospital long-term anticoagulation management, patients are referred to dedicated 
anticoagulation clinics with the aim of maintaining a stable therapeutic INR level. We follow a standard, uniform, and validated protocol of long-term postprocedural anticoagulation management [16]. Briefly, oral anticoagulation is discontinued, regardless of the $\mathrm{CHADS}_{2}$ score, if patients do not experience any recurrence of atrial tachyarrhythmias, severe pulmonary vein stenosis (pulmonary vein narrowing $>70 \%$ ), and severe left atrial mechanical dysfunction, as assessed by transthoracic echocardiography.

Patients with a $\mathrm{CHADS}_{2}$ score $\geq 1$ experiencing early recurrence of $\mathrm{AF}$ are maintained on warfarin for at least 6 months. In these patients, warfarin is discontinued if there is no $\mathrm{AF}$ recurrence in the last 3 months without antiarrhythmic drugs, and aspirin 81 to $325 \mathrm{mg}$ is started. In case of new AF recurrence after warfarin discontinuation in patients with a $\mathrm{CHADS}_{2}$ score $\geq 1$, oral anticoagulation is restarted.

\section{Discussion}

Our approach to periprocedural anticoagulation has been extensively validated in previous work $[12,13,17]$. In particular, we found that a conventional anticoagulation approach, which included warfarin discontinuation with peri- and postprocedural bridging with unfractioned and low-molecular-weight heparin actually increases the risk of bleeding and thromboembolic complications, as compared to no periprocedural interruption of oral anticoagulation $[12,13,17]$.

Our most recent report provides strong evidence that performing AF ablation under therapeutic INR is a safe and effective approach to virtually abolish the risk of thromboembolic complications [13]. We reported a multicenter prospective comparison of three anticoagulation protocols in 9 centers performing the same ablation procedure. A total of 6,454 patients were included in the study, of whom 2,488 underwent ablation with an $8-\mathrm{mm}$ ablation catheter and preprocedural warfarin discontinuation (Group 1), 1,348 underwent ablation with an open irrigated catheter and preprocedural warfarin discontinuation (Group 2), and 2,618 underwent ablation with an open irrigated catheter without preprocedural warfarin discontinuation (Group 3). Overall, periprocedural thromboembolic complications occurred in $39(0.6 \%)$ patients, with a rate of $1.1 \%$ in Group 1 , and of $0.9 \%$ in Group 2. Notably, no patient of Group 3 experienced periprocedural thromboembolism (Figure 1). These data support also the appropriateness of our approach to TEE based on the intensity of anticoagulation in the month preceding the procedure, especially considering that 1,178 (45\%) Group 3 patients had persistent AF, and 498 (19\%) long-standing persistent AF.

At multivariable analysis, which adjusted for age, gender, coronary artery disease, type of AF, heart failure, diabetes, hypertension, and prior stroke, the anticoagulation strategy of ablation with a therapeutic INR was a strong independent predictor of lower periprocedural thromboembolic events (odds ratio $0.54,95 \%$ confidence interval 0.32 to 0.89, $P=.017)$. With regard to bleedings, the pooled

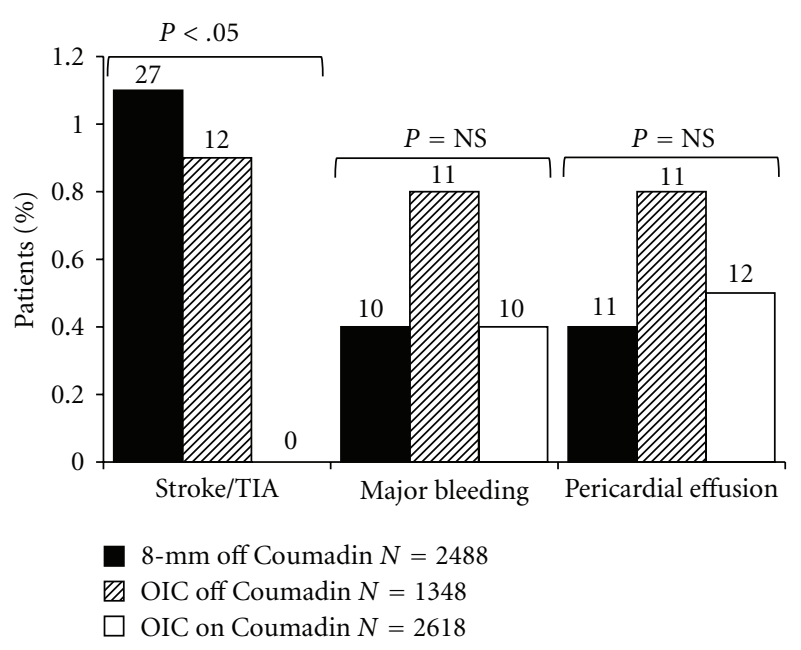

FIGURE 1: Complications of radiofrequency catheter ablation of atrial fibrillation in 6,454 patients referred to our institution between 2002 and 2009. TIA = transient ischemic attack; 8-mm $=8-\mathrm{mm}$ nonirrigated ablation catheter; $\mathrm{OIC}=$ open irrigated ablation catheter; off/on Coumadin = periprocedural discontinuation/maintenance of therapeutic oral anticoagulant therapy with warfarin (Coumadin). $P$ from multiple comparison between OIC on Coumadin and 8-mm and OIC off Coumadin: data from Di Biase et al. [13].

rate of major bleeding complications (i.e., bleeding requiring interventions including transfusions, hemopericardium, hemothorax, and retroperitoneal bleeding) and pericardial effusion in patients who discontinued warfarin before the ablation procedure (Groups 1 and 2) was 1.1\%, whereas in Group 3 was $0.8 \%$ (Figure 1). If also minor bleedings were included, patients who discontinued warfarin before ablation procedure had a pooled rate of bleeding complications of $20.7 \%$, while patients who were maintained on warfarin had a rate of $4.8 \%$.

Translating such percentages into treatment effects, the net clinical benefit associated with AF ablation without interruption of oral anticoagulation is overwhelming (Figure 2), with an estimated 170 thromboembolic or hemorrhagic complications avoided every 1,000 patients ablated.

In the most recent survey on AF catheter ablation, Cappato et al. reported thromboembolic and hemorrhagic complication rates in 16,039 patients undergoing AF ablation between 2003 and 2006 in 521 centers distributed worldwide [18]. All these patients had oral anticoagulant discontinuation before the ablation procedure. Accordingly, thromboembolic and bleeding complication rates in this large survey were fairly consistent with that reported in Group 1 and 2 patients of our study $[13,18]$. Based on our findings it is estimable that, if periprocedural oral anticoagulation was not discontinued in all patients included in the survey, more than 2,700 thromboembolic or hemorrhagic complications would have been avoided worldwide from 2003 to 2006 . Therefore, there are cogent data supporting the benefit of AF ablation without discontinuation of oral anticoagulation. 


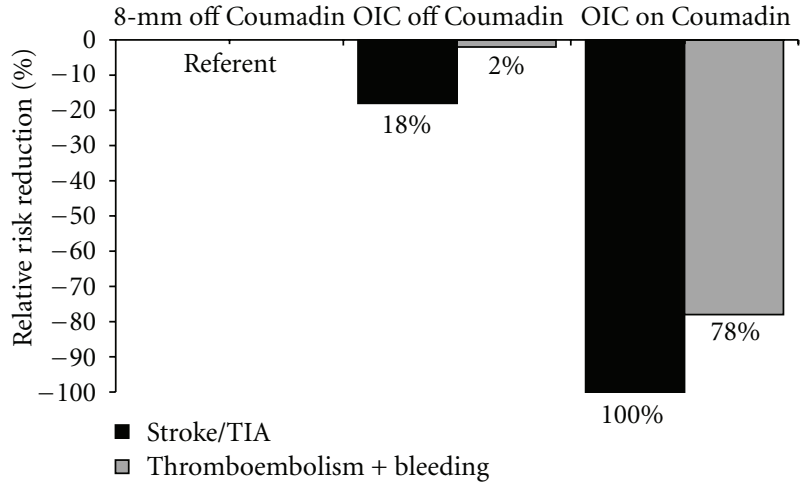

FIGURE 2: Benefits of atrial fibrillation ablation without interruption of oral anticoagulation. TIA $=$ transient ischemic attack; 8$\mathrm{mm}$ off Coumadin (referent group) $=8-\mathrm{mm}$ nonirrigated ablation catheter with periprocedural discontinuation of therapeutic oral anticoagulant therapy with warfarin (Coumadin); OIC off Coumadin $=$ open irrigated ablation catheter with periprocedural discontinuation of therapeutic oral anticoagulant therapy with Coumadin; OIC on Coumadin = open irrigated ablation catheter with periprocedural maintenance of therapeutic oral anticoagulant therapy with Coumadin: data from Di Biase et al. [13].

Since the acute reversal of the anticoagulant effect of warfarin is possible only through infusion of fresh coagulation factors, we routinely type- and cross-match all patients, so that packed red blood cells and fresh frozen plasma are readily available for infusion in case of hemorragic complications. If the preprocedural INR is above 3.5, we partially reverse the anticoagulant effect with one to two units of fresh frozen plasma.

Of interest, the strategy of ablation under therapeutic INR could also be more cost-effective compared to bridging therapy with enoxaparin, which is expensive and may be inconvenient for many patients.

\section{Conclusions}

Radiofrequency catheter ablation of AF without discontinuation of oral anticoagulation significantly reduces the risk of thromboembolic and minor bleeding complications. Maintenance of periprocedural therapeutic INR should be considered the anticoagulation strategy of choice among patients submitted to catheter ablation of AF.

\section{References}

[1] Y. Miyasaka, M. E. Barnes, B. J. Gersh et al., "Secular trends in incidence of atrial fibrillation in Olmsted County, Minnesota, 1980 to 2000, and implications on the projections for future prevalence," Circulation, vol. 114, no. 2, pp. 119-125, 2006.

[2] J. Friberg, P. Buch, H. Scharling, N. Gadsbøll, and G. B. Jensen, "Rising rates of hospital admissions for atrial fibrillation," Epidemiology, vol. 14, no. 6, pp. 666-672, 2003.

[3] P. A. Wolf, R. D. Abbott, and W. B. Kannel, "Atrial fibrillation as an independent risk factor for stroke: the Framingham Study," Stroke, vol. 22, no. 8, pp. 983-988, 1991.
[4] E. J. Benjamin, P. A. Wolf, R. B. D'Agostino, H. Silbershatz, W. B. Kannel, and D. Levy, "Impact of atrial fibrillation on the risk of death: the Framingham Heart Study," Circulation, vol. 98, no. 10, pp. 946-952, 1998.

[5] A. Natale, A. Raviele, T. Arentz et al., "Venice chart international consensus document on atrial fibrillation ablation," Journal of Cardiovascular Electrophysiology, vol. 18, no. 5, pp. 560-580, 2007.

[6] R. Cappato, H. Calkins, S. A. Chen et al., "Worldwide survey on the methods, efficacy, and safety of catheter ablation for human atrial fibrillation," Circulation, vol. 111, no. 9, pp. 1100-1105, 2005.

[7] S. Dixit and F. E. Marchlinski, "How to recognize, manage, and prevent complications during atrial fibrillation ablation," Heart Rhythm, vol. 4, no. 1, pp. 108-115, 2007.

[8] L. C. Kok, J. M. Mangrum, D. E. Haines, and J. P. Mounsey, "Cerebrovascular complication associated with pulmonary vein ablation," Journal of Cardiovascular Electrophysiology, vol. 13, no. 8, pp. 764-767, 2002.

[9] D. Scherr, K. Sharma, D. Dalal et al., "Incidence and predictors of periprocedural cerebrovascular accident in patients undergoing catheter ablation of atrial fibrillation," Journal of Cardiovascular Electrophysiology, vol. 20, no. 12, pp. 13571363, 2009.

[10] S. Connolly, J. Pogue, R. Hart et al., "Clopidogrel plus aspirin versus oral anticoagulation for atrial fibrillation in the atrial fibrillation clopidogrel trial with irbesartan for prevention of Vascular Events (ACTIVE W): a randomised controlled trial," Lancet, vol. 367, no. 9526, pp. 1903-1912, 2006.

[11] H. Calkins, J. Brugada, D. L. Packer et al., "HRS/EHRA/ECAS Expert Consensus Statement on Catheter and Surgical Ablation of Atrial Fibrillation: recommendations for personnel, policy, procedures and follow-up. A report of the Heart Rhythm Society (HRS) task force on catheter and surgical ablation of atrial fibrillation. Developed in partnership with the European Heart Rhythm Association (EHRA)," Heart Rhythm, vol. 4, no. 6, pp. 816-861, 2007.

[12] O. M. Wazni, S. Beheiry, T. Fahmy et al., "Atrial fibrillation ablation in patients with therapeutic international normalized ratio: comparison of strategies of anticoagulation management in the periprocedural period," Circulation, vol. 116, no. 22, pp. 2531-2534, 2007.

[13] L. Di Biase, J. D. Burkhardt, P. Mohanty et al., "Periprocedural stroke and management of major bleeding complications in patients undergoing catheter ablation of atrial fibrillation: the impact of periprocedural therapeutic international normalized ratio," Circulation, vol. 121, no. 23, pp. 2550-2556, 2010.

[14] M. H. Kanj, O. M. Wazni, and A. Natale, "How to do circular mapping catheter-guided pulmonary vein antrum isolation: the Cleveland Clinic approach," Heart Rhythm, vol. 3, no. 7, pp. 866-869, 2006.

[15] A. Bulava, L. Slavík, M. Fiala et al., "Endothelial damage and activation of the hemostatic system during radiofrequency catheter isolation of pulmonary veins," Journal of Interventional Cardiac Electrophysiology, vol. 10, no. 3, pp. 271-279, 2004.

[16] S. Themistoclakis, A. Corrado, F. E. Marchlinski et al., "The risk of thromboembolism and need for oral anticoagulation after successful atrial fibrillation ablation," Journal of the American College of Cardiology, vol. 55, no. 8, pp. 735-743, 2010 . 
[17] A. A. Hussein, D. O. Martin, W. Saliba et al., "Radiofrequency ablation of atrial fibrillation under therapeutic international normalized ratio: a safe and efficacious periprocedural anticoagulation strategy," Heart Rhythm, vol. 6, no. 10, pp. 14251429, 2009.

[18] R. Cappato, H. Calkins, S. A. Chen et al., "Updated worldwide survey on the methods, efficacy, and safety of catheter ablation for human atrial fibrillation," Circulation, vol. 3, no. 1, pp. 32$38,2010$. 


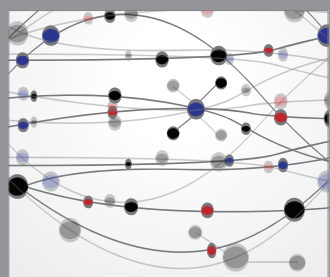

The Scientific World Journal
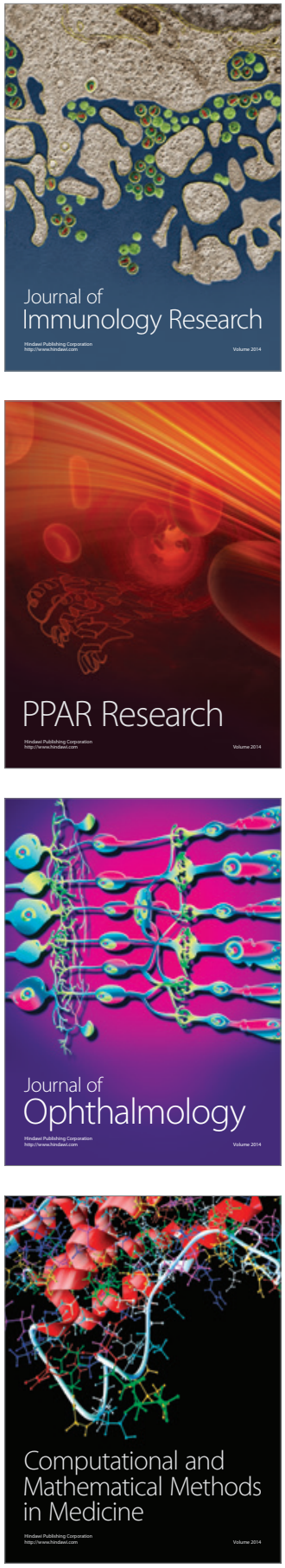

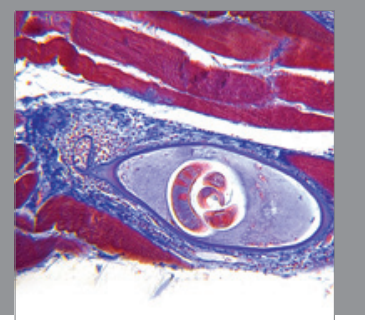

Gastroenterology

Research and Practice
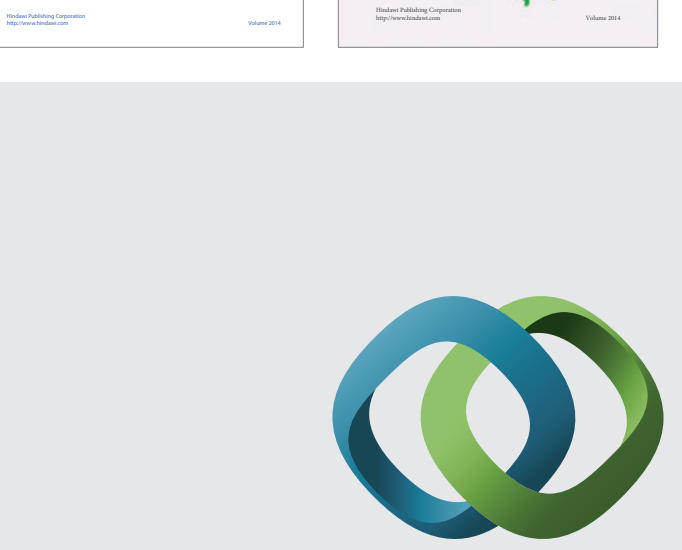

\section{Hindawi}

Submit your manuscripts at

http://www.hindawi.com
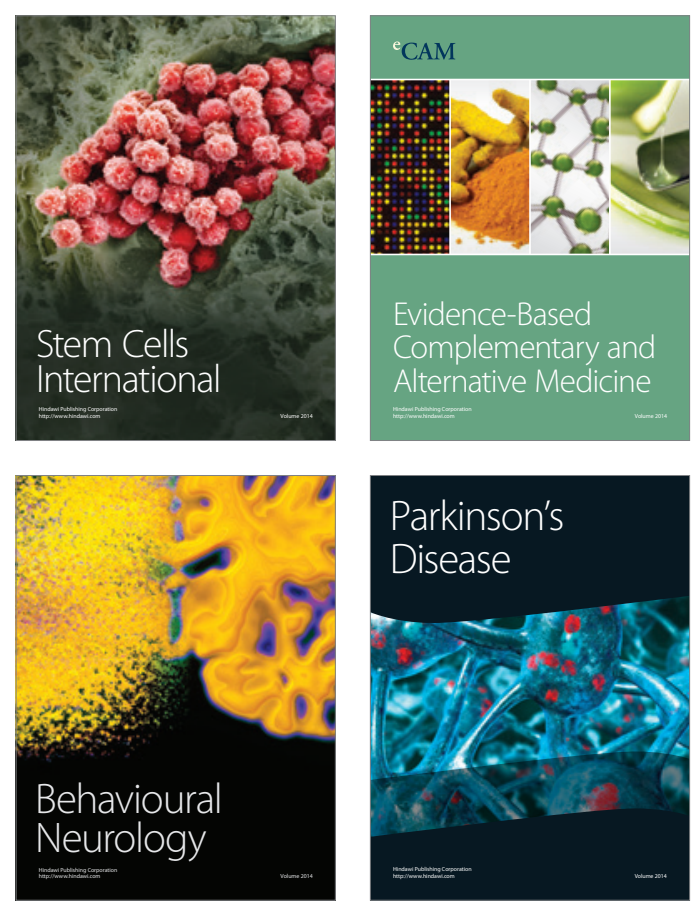

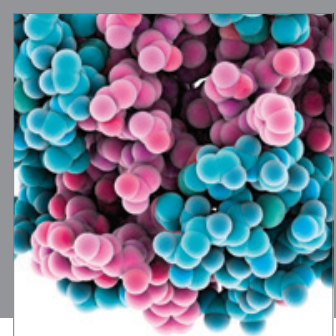

Journal of
Diabetes Research

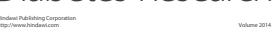

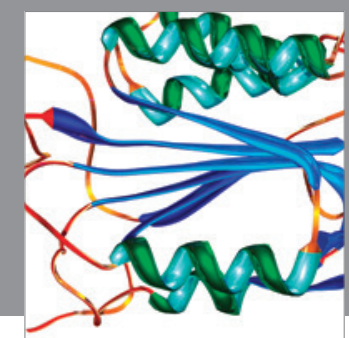

Disease Markers
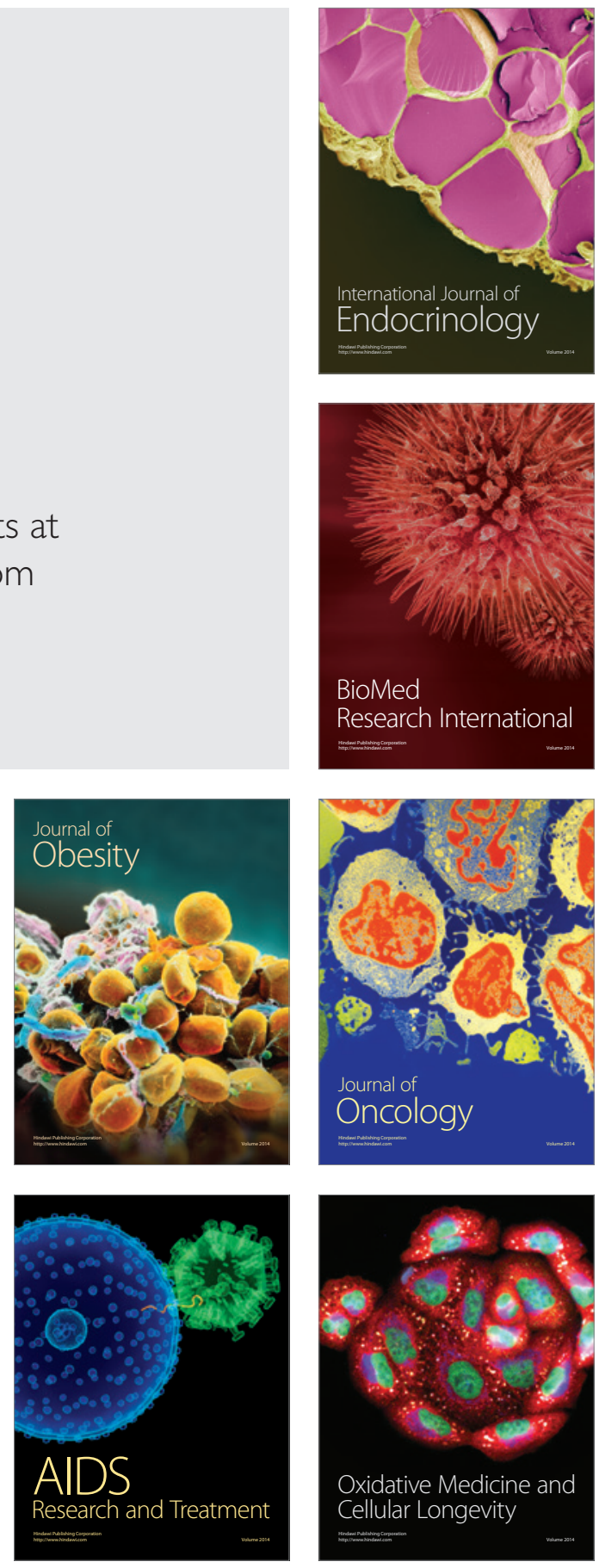